Revue d'histoire de l'enfance « irrégulière »

Le Temps de l'histoire

12 | 2010

Autour de l'enfant : la ronde des professionnels

\title{
Présentation du dossier
}

\section{Samuel Boussion}

\section{(2) OpenEdition \\ Journals}

Édition électronique

URL : http://journals.openedition.org/rhei/3183

DOI : 10.4000/rhei.3183

ISBN : 978-2-7535-1651-9

ISSN : 1777-540X

Éditeur

Presses universitaires de Rennes

Édition imprimée

Date de publication : 30 novembre 2010

Pagination : 5-20

ISBN : 978-2-7535-1259-7

ISSN : 1287-2431

Référence électronique

Samuel Boussion, «Présentation du dossier », Revue d'histoire de l'enfance «irrégulière » [En ligne],

12 | 2010, mis en ligne le 30 novembre 2012, consulté le 22 avril 2019. URL : http://

journals.openedition.org/rhei/3183; DOI : 10.4000/rhei.3183

Ce document a été généré automatiquement le 22 avril 2019

(c) PUR 


\title{
Présentation du dossier
}

\author{
Samuel Boussion
}

1 À sa manière, le précédent numéro de la Revue d'histoire de l'enfance «irrégulière » a déjà rendu compte de l'agrégation progressive d'intervenants au chevet de l'enfant difficile au cours $\mathrm{du} \mathrm{XX}^{\mathrm{e}}$ siècle, que ce dernier relève de l'Assistance publique, de la Justice ou d'établissements médicopédagogiques, d'observation, etc. ${ }^{1}$ Les dossiers individuels élaborés au cœur des institutions ont été ainsi la mise par écrit d'une partition jouée par plusieurs professionnels. Ceux-là représentent alors autant de regards objectivant l'enfant, préoccupés de sa personnalité mais aussi de ses antécédents, de sa famille, plus largement de son milieu, en somme son «casier social $»^{2}$. Le dossier est ainsi un réceptacle formalisé des diverses pratiques professionnelles encadrant l'enfant aux prises avec les institutions ou la Justice, dans le but de dresser une généalogie explicative de ce qui l'a amené en ces mains savantes et de lui tracer un devenir, donnant lieu à une accumulation de pièces, souvent redondantes ${ }^{3}$, qui sont autant de traces de cette activité experte : jugements et ordonnances, rapports, enquête sociale, notes de comportement, jusqu'aux écrits demandés aux jeunes eux-mêmes... ${ }^{4}$

2 C'est que depuis le XIX siècle, à mesure que l'intérêt et le souci pour l'enfance se sont construits ${ }^{5}$, en même temps que la ségrégation de ses tranches les plus problématiques, de nombreux personnages se sont pressés autour de l'enfant, qui ont cherché à l'instruire, l'éduquer, le juger, le soigner, le panser, l'observer, le comprendre... Le XIX ${ }^{\text {e }}$ siècle fut celui de la constitution du champ de la protection de l'enfance, qui renvoie luimême à une délimitation par strates successives, selon les établissements, les politiques, la législation, mais aussi les acteurs. De nombreux travaux ont en effet permis de souligner, à ce moment donné, la sollicitude de plus en plus grande de la communauté nationale envers les enfants, aussi bien sous ses aspects protecteurs que répressifs, l'élargissement des catégories d'enfants pris en charge ${ }^{6}$, ainsi que des objectifs de l'intervention (vers une éducation morale) ${ }^{7}$. Aussi, cette objectivation de l'enfance, souvent à partir de ses déviances, est indissociable des dynamiques professionnelles à l'œuvre dans ce champ, depuis les philanthropes du XIX siècle jusqu'aux formes plus professionnalisées du siècle suivant. Partant du constat qu'il n'existe pas de travail de 
synthèse qui relie ces différentes professions, ce dossier de la Revue d'histoire de l'enfance "irrégulière» se justifie en ce qu'il se propose de dresser un état des connaissances, de lancer des pistes de recherche, de questionner des professions peu ou pas étudiées jusque-là, mais aussi de comparer entre elles des dynamiques professionnelles, parfois convergentes, parfois concurrentes, en somme d'étudier des processus de professionnalisation, ceux-ci étant en effet fondamentaux pour comprendre l'évolution des institutions, des politiques ainsi que des pratiques.

3 Les professions méritaient d'être évoquées en tant que telles, sous la forme de monographies. Certaines de ces professions sont plus visibles, parce que plus prestigieuses, comme des archétypes de ce que l'on nomme " professions » (les médecins notamment). Elles sont aussi mieux instituées et détiennent une forme de pouvoir conféré par leur position dans la chaîne du traitement de l'enfance irrégulière, ce que rappelle avec autorité le pédopsychiatre Georges Heuyer en 1953, dans la préface d'une édition des guides Néret consacrée aux "métiers de l'éducation difficile ${ }^{8}$. Faisant le point sur l'éventail des métiers, on y retrouve évoquées - et dans cet ordre - les figures du pédopsychiatre, du juge des enfants, du délégué permanent à la liberté surveillée, de l'assistante sociale auprès des tribunaux, du psychologue et du psychotechnicien, de l'éducateur de jeunes inadaptés et enfin des maîtres de formation scolaire et professionnelle. Pour se justifier de cette hiérarchisation, Georges Heuyer insiste sur le rôle central du pédopsychiatre dans le dépistage et le traitement de l'enfance inadaptée :

«Si le livre est un exposé de l'ensemble de l'inadaptation dont la délinquance infantile n'est qu'un aspect épisodique, c'est au médecin que l'enfant est amené d'abord et il appartient au médecin psychiatre d'exposer sa conception du travail d'équipe dont il est le centre et dont il a la direction. $"^{9}$

La plupart de ceux que nous avons appelés ici «professionnels» relèvent en fait davantage de segments qui s'inscrivent dans des corps plus larges: selon Jean-Christophe Coffin, les psychiatres de l'enfance doivent se faire une place parmi les autres psychiatres, voire parmi les médecins en général ; les assistantes sociales étudiées par Pascale QuincyLefebvre interviennent dans le cadre de services sociaux « spécialisés », etc. Dès lors, on comprend mieux la quête de légitimité et de reconnaissance de ces acteurs vis-à-vis des pouvoirs publics mais aussi des autres professions, derrière une forme de "geste" professionnelle qui leur serait propre : l'enquête sociale chez les assistantes sociales ou la guidance infantile chez certains psychiatres. Dans ces deux cas, l'après seconde guerre mondiale est une période propre au renouvellement de leur professionnalité, entendue comme ensemble des compétences définissant une profession, dans une volonté de rupture et de refondation; c'est ainsi que l'on peut lire la redécouverte du casework par les assistantes sociales ou les diverses démarcations des psychiatres d'avec la doctrine dominante dite des « constitutions » qui ont eu cours depuis l'entre-deux-guerres.

Un autre intérêt de cet éventail des professions est de lever le voile sur certaines qui sont restées longtemps des angles morts de la recherche sur l'histoire de l'enfance irrégulière, sans doute parce qu'elles ont tout autant représenté des figures impensées dans les dispositifs de protection de l'enfance. Pourtant, avocats de mineurs et policiers - puisqu'il s'agit d'eux - occupent une part non négligeable de la scène dans laquelle évoluent les mineurs. On rencontre les premiers au tribunal pour enfants tandis que les seconds se croisent au coin de la rue ou au commissariat. Le travail de Patricia Benech-Le Roux réactualise ses recherches antérieures sur les avocats en prolongeant sa réflexion sur des temporalités jusque là peu explorées. Présents sur la scène judiciaire dès la fin du XIX ${ }^{e}$ 
siècle, notamment par le biais de leur puissant investissement au sein des comités d'enfants traduits en justice, ces professionnels de la parole vont devenir progressivement des acteurs «muets» de la justice des mineurs, leur légitimité étant remise en cause tandis que d'autres professionnels interviennent déjà au service de l'appareil judiciaire pour éclairer les décisions du juge. Mathias Gardet, quant à lui, ressuscite la brigade des mineurs des années 1950-1960, et plus largement une partie des actions spécifiques de la police en matière de prévention de la délinquance juvénile. Loin d'être dans la seule confrontation et le maintien de l'ordre, leur connaissance du « terrain » et les formations qu'ils suivent en font au contraire des acteurs intégrés à part entière dans cette partition.

6 Une scansion importante de l'histoire de ces professions est celle de l'immédiat aprèsguerre. Dans ce contexte de reconstruction propre aux utopies et aux réformes, de nouvelles professionnalités s'esquissent. Les psychiatres de l'enfance souhaitent ainsi faire rupture après le désastre de la psychiatrie pendant la guerre et l'occupation. Mais l'un des apports les plus importants de cette période qui s'ouvre après 1945 est l'apparition sur la scène de nouveaux acteurs: les éducateurs. Déjà en germe dans quelques textes programmatiques des années 1930, ils ne deviendront en France des professionnels de chair que dans la décennie suivante ${ }^{10}$. Encore faudrait-il ici parler de deux branches distinctes, au moins dans cet exemple français, qui distinguent éducateurs de l'Éducation surveillée puis de la Protection judiciaire de la jeunesse (PJJ), objets de l'article de Ludovic Jamet, dotés d'un statut dès 1946 et dépendants des politiques menées par le ministère de la Justice, et des éducateurs appelés au tournant des années 1950 "éducateurs spécialisés ", intervenant dans un champ plus vaste et constituant une profession en perpétuelle construction.

7 Un autre apport de ces contributions se situe dans l'attention portée envers les institutions où circulent les différents modèles, notamment au niveau transnational. Dès le $\mathrm{XIX}^{\mathrm{e}}$ siècle, les voyages à l'étranger ainsi que les premiers congrès internationaux ont contribué à de multiples échanges qui ont permis des importations et surtout des adaptations d'expériences. Le $\mathrm{XX}^{\mathrm{e}}$ siècle ne déroge pas à cette règle, tout en semblant l'accentuer. Psychiatres, assistantes sociales viennent puiser dans les expérimentations anglo-saxonnes, tandis que l'on aurait pu souligner dans le même temps les efforts similaires de quelques éducateurs français de l'après-guerre pour adapter dans quelques institutions le group-work et le sociodrame, made in USA ${ }^{11}$. La pratique policière en matière de délinquance juvénile et de prévention, selon Mathias Gardet, tirera aussi largement profit des rencontres internationales dans les années 1950, l'impact en étant repérable en partie dans les changements de doctrine et dans l'investissement de quelques officiers. Mais surtout, à une période où se consolident des organisations transnationales dans la foulée de la reconstruction et du Plan Marshall, que l'on pense à l'UNESCO notamment, l'un des faits marquants est le rôle joué par des groupements tels que celui observé par Louise Bienvenue et Lucia Ferretti, le BICE (Bureau international catholique de l'enfance) dans les années 1950-1960. Cette perspective ouvre de multiples portes vers la connaissance d'autres réseaux qui se forgent à la même période à l'échelon international et qui contribuent à de multiples échanges, aussi bien sur le plan des discours que des savoirs, dont les effets sur les politiques de protection de l'enfance ainsi que sur les pratiques professionnelles doivent être mesurés. est un axe privilégié par la plupart des contributions. Elle est d'abord repérable à travers 
la place faite aux groupements professionnels, par exemple la FNSSS (Fédération nationale des services sociaux spécialisés), l'ANAS (Association nationale des assistantes sociales), jusqu'aux groupes d'avocats de mineurs des années 1990, qui sont effectivement des opérateurs pertinents de la compréhension des processus de professionnalisation. D'abord parce que ces organisations effectuent en effet un travail de définition et de délimitation du groupe socioprofessionnel en explicitant constamment ce que doit être la profession; aussi parce qu'elles sont en quête de reconnaissance sociale et institutionnelle, voire de monopole et de protection dans l'exercice du métier; enfin par le concours qu'elles prêtent à la technicisation de la pratique professionnelle, à la structuration du savoir et à la définition de normes professionnelles ${ }^{12}$.

9 La construction des identités professionnelles et la recherche de légitimité se révèlent aussi dans les sources utilisées ici par les auteurs. Les corpus manipulés sont essentiellement composés d'articles de périodiques professionnels, moins souvent de littérature grise. En effet, chacun semble vouloir affirmer la pertinence de son activité, les ressorts de sa pratique ainsi que les savoirs sur lesquels elle repose. C'est aussi une manière de faire rupture avec des représentations traditionnelles ; ainsi les policiers qui mettent en avant leur rôle dans la prévention, «ni croquemitaines, ni matraqueurs ». L'après Seconde Guerre mondiale est à ce titre une période importante, où l'on constate une profusion d'écrits, les revues spécialisés fleurissant à quelques années d'intervalles, car «une politique s'élabore, qui suscite de l'écriture " ${ }^{13}$. Chacune occupe un espace délimité au sein d'un champ qui a du mal à s'unifier et parfois, elles se concurrencent ; l'exemple des psychiatres est assez éloquent sur ce point après la Libération, entre L'Évolution psychiatrique, Psychiatrie de l'enfant ou la Revue de neuropsychiatrie infantile et d'hygiène mentale de l'enfance, où chaque revue incarne une forme de courant à l'intérieur de la discipline.

10 A certains égards, ce numéro est ancré dans les réalités de la pratique professionnelle contemporaine et dans les recompositions des professions, à l'œuvre depuis peu ${ }^{14}$. La place accordée au regard de sociologues sur les professions témoigne justement de la persistance des doutes identitaires identifiables depuis les années 1990. Le retour à une politique plus "pénale » en matière de justice des mineurs a engendré semble-t-il des évolutions importantes, à la fois pour les avocats, en quelque sorte à nouveau légitimés, mais aussi pour les policiers et surtout pour les éducateurs de la Protection judiciaire de la jeunesse, dont la professionnalisation leur semble aller à rebours des valeurs sur lesquelles repose leur relation au métier, qu'ils estiment attachées à l'esprit de l'ordonnance de $1945^{15}$. Ces questionnements issus de la pratique quotidienne expliquent aussi la prégnance d'un regard sociologique sur les groupes professionnels, depuis longtemps sensible à la fois au développement des idéologies professionnelles et aux situations de travail, mais qui se fait parfois au détriment d'une approche historique.

$11 \mathrm{Au}$ moment de conclure, certains regrets pointent. D'abord que la quasi-totalité des contributions concernent exclusivement la France, à l'exception d'une incursion au Québec. Ensuite, malgré des appels du pied, quelques acteurs importants ont été laissés de côté, sans doute faute de recherches actuelles dans ce domaine. Que l'on pense aux catégories de personnel des établissements spécialisés, dont il est trop peu fait mention : instituteurs, éducateurs chargés de classe, puis éducateurs scolaires; moniteurs techniques ou professionnels, professeurs ou instructeurs techniques, plus tard éducateurs techniques; et quid des infirmières, des aumôniers, des hommes à tout faire... Certaines recherches sont en cours, qui éclaireront l'histoire des juges des enfants, celle 
des délégués à la liberté surveillée. D’autres pistes auraient pu être suivies, par exemple celle de l'histoire des formations. Quoiqu'il en soit, ce numéro est un marchepied vers d'autres recherches. D'ores et déjà, dans l'optique de réunir ces professions et métiers dans une approche diachronique, une exposition sera réalisée à l'horizon fin 2011, selon une scénographie adaptée à son objet: galerie de portraits, représentations de professionnels, extraits radiophoniques et télévisés, textes fondamentaux, revues professionnelles, etc.

\section{NOTES}

1. "Paroles libres, paroles captives », dossier sous la direction de Mathias Gardet, Revue d'histoire de l'enfance "irrégulière », nº 11, octobre 2009.

2. Philippe meyer, L'enfant et la raison d'Etat, Paris, Seuil, 1977, p. 114.

3. Jacques Donzelot n'hésitera pas à parler de « moutonnement du même discours ", voir : Jacques Donzelot, La police des familles, Paris, éd. de Minuit, 1977, p. 109.

4. Voir Samuel Boussion, Mathias Gardet, «Entre ombre et lumière. Les dossiers de mineurs en Justice au regard de la conservation, de la recherche et des pratiques professionnelles", Archives de France, La protection de l'enfance. Ecrits protégés, écrits ignorés. Les dossiers individuels de mineurs et de jeunes majeurs sous main de justice, Paris, La Documentation française, 2010, p. 119-129.

5. Michelle Perrot, "Sur la notion d'intérêt de l'enfant et son émergence au XIX siècle ", Actes, $n^{\circ} 37$, juin 1982, p. 40-43.

6. Catherine Rollet-Echallier, La politique à l'égard de la petite enfance sous la III République, 1865-1939, Paris, PUF, 1990, 677 p. ; Jean-Pierre в ardet, Jean-Noël Luc, Isabelle R obin-R omero, Catherine Rollet (dir.), Lorsque l'enfant grandit. Entre dépendance et autonomie, Paris, Presses de l'université de Paris Sorbonne, 2003, 983 p.

7. Dominique Dessertine, «L'émergence de la politique sociale de l'enfance: des enfants trouvés à l'enfance assistée 1780-1940 ", Vie sociale, « Le social aux prises avec l'histoire », $\mathrm{n}^{\circ} 3 / 4,1990$.

8. Jean chazAL, Andrée Dauphin, Henri joubrel (dir.), Les métiers de l'éducation difficile, Paris, éd. Lamarre, coll. Guides Néret, 1953, 115 p.

9. Ibid., p. 8.

10. Samuel BOUSSION, Les éducateurs spécialisés et leur association professionnelle: l'ANEJI (Association nationale des éducateurs de jeunes inadaptés) de 1947 à 1967. Naissance et construction d'une profession sociale, thèse de doctorat en histoire, université d'Angers, 2007, 3 vol.

11. Samuel Boussion, «Made in USA ou quand le sociodrame vint à Vitry (années 1950)", communication lors de la journée d'étude sur le foyer de semi-liberté de Vitry-sur-Seine, $1^{\text {er }}$ avril 2010. 
12. Jean RUHLMANN, «Identité professionnelle et indépendance dans les professions mobilisées dans la défense des classes moyennes sous le Front populaire ", Pierre guillaume (dir.), La professionnalisation des classes moyennes, Talence, éd. de la Maison des sciences de l'homme d'Aquitaine, 1996, p. 343.

13. Françoise TÉTARD, «Après 1945 : partage de pouvoirs ou complémentarité ", Informations sociales, $\mathrm{n}^{\circ} 53$, « Social et médias », 1996, p. 20.

14. Voir à ce sujet Sociétés et jeunesses en difficulté, dossier «La construction de la professionnalité éducative $", \mathrm{n}^{\circ} 7$, printemps 2009 [en ligne].

15. A ce titre, l'article de Ludovic Jamet est à rapprocher de: Nicolas SALLÉE, «Les éducateurs de la Protection judiciaire de la jeunesse à l'épreuve de l'évolution du traitement pénal des jeunes délinquants ", Champ pénal / Penal field, nouvelle revue internationale de criminologie, Vol. VII, 2010.

\section{AUTEUR}

\section{SAMUEL BOUSSION}

Historien, maître de conférences en sciences de l'éducation, CIRCEFT, université Paris 8. 